\title{
Substance Use Pattern among Nursing Students in Jos, North Central Nigeria
}

\author{
Nwoga Charles ${ }^{*}$, Ndak Zuhumnan², Danboyi Mafai ${ }^{3}$, Dakwak Samuel ${ }^{4}$, Piwuna Christopher ${ }^{1}$, \\ Armiyau Aishetu ${ }^{5}$
}

\author{
${ }^{1}$ Department of Psychiatry, University of Jos, Jos, Plateau State, Nigeria \\ ${ }^{2}$ Hospital Management Board, Jos, Plateau State, Nigeria \\ ${ }^{3}$ Quintessential Healthcare Centre, Jos, Plateau State, Nigeria \\ ${ }^{4}$ Department of Psychology, University of Jos, Jos, Plateau State, Nigeria \\ ${ }^{5}$ Jos University Teaching Hospital, Jos, Plateau State, Nigeria \\ Email: *nwogacharles@gmail.com, *nwogac@unijos.edu.ng
}

How to cite this paper: Charles, N., Zuhumnan, N., Mafai, D., Samuel, D., Christopher, P. and Aishetu, A. (2021) Substance Use Pattern among Nursing Students in Jos, North Central Nigeria. Open Journal of Psychiatry, 11, 80-90.

https://doi.org/10.4236/ojpsych.2021.112008

Received: January 26, 2021

Accepted: April 13, 2021

Published: April 16, 2021

Copyright $\odot 2021$ by author(s) and Scientific Research Publishing Inc. This work is licensed under the Creative Commons Attribution International License (CC BY 4.0).

http://creativecommons.org/licenses/by/4.0/

\section{Open Access}

\begin{abstract}
Aim: To establish the substance use pattern and psychological distress among the in-coming nursing students of the College with a view towards recommending ways to prevent or reduce complications of substance use disorder in the college. Study design: A cross-sectional design was used. Place and duration: The study took place at the College of Nursing and Midwifery, Jos, Plateau state between the $3^{\text {rd }}$ and $14^{\text {th }}$ of February, 2020. Methodology: The study was carried out during the orientation program of the 206 first-year students of the college. Following approval, a health talk and explanation of the aims and objectives, their consent was obtained in writing. Two-staged sampling with a sociodemographic questionnaire and Hopkins Symptom Checklist- 25 followed by urine drug testing was done. The participants were debriefed on the findings while maintaining strict confidentiality. There were 65 males and 141 females. Results: Lifetime prevalence rate of any substance use was $21.8 \%$. More females 33 (23.4\%) than males 12 (18.5\%) used substances. Only 9 (4.5\%) volunteered their substance use, the rest was revealed by the urine testing. Prevalence of alcohol was 23 (11.2\%), ketamine 13 (6.3\%), nicotine $3(1.5 \%)$, benzodiazepines $3(1.5 \%)$, cannabis $2(1.0 \%)$ and opiods $1(0.5 \%)$. Symptoms of psychological distress were high as $118(57.3 \%)$ and $82(38.8 \%)$ were symptomatic for anxiety and depression respectively. Alcohol use was significantly related with having symptomatic anxiety $(P=$ $0.02)$ and depression $(P=0.00)$ in the study. Conclusion: Considering the high prevalence of substance use among the participants, educational intervention and policies should be designed to prevent substance dependence with its complications within the students.
\end{abstract}




\section{Keywords}

Substance Use, Students, Jos, Nigeria

\section{Introduction}

Substance use among young people is both a clinical and public health issue. It is usually associated with academic, psychological, social, behavioral as well as physical problems. Consumption of licit and illicit drugs has increased in around the world, including Nigeria. People use substances for various reasons. College period can and should be an exciting and enriching experience. To help ensure that it will be students and their parents will want to choose a college that fosters students' academic and social development and promotes campus and community safety. One critical element to consider is the alcohol and other drug culture of a campus. Far too often, alcohol and other drug abuse result in tragedy [1]. Among all populations, college students are one of the groups in danger of substance abuse [2]. College is a critical period that young people can commence the use of substances. Reasons include academic pressure, unlimited freedom, peer groups and easy access to common substances like alcohol and cigarette. Substance use often progresses to a disorder and has contributed to poor performance among students. Although the majority of college students avoid the unsafe use of alcohol and other drugs, they are still likely to suffer the consequences of the high-risk behaviors of their peers [1]. College students encounter problems when other colleagues are high on drugs. They have to deal with interruption of their studies and sleep, have to take care of a drunken colleague, insulted or humiliated, have serious arguments or quarrel, or even be pushed, hit or assaulted [3]. Alcohol and other drug abuse are a factor in the majority of accidents, injuries, vandalism, and crime on college campuses and is frequently a key factor when students encounter problems with their course work [3] [4]. A substance abuser is 18 times more likely to be involved in criminal activity than someone in the general population [5].

Alcohol use is a major risk factor for mortality and morbidity, playing a role in more than 200 diseases and injury outcomes [6]. Students who engage in high-risk drinking and other drug use are not just harming themselves but also those around them, and not just occasionally but with truly disturbing frequency [7]. Alcohol is strongly associated with both intentional and unintentional injury [5]. Smoking also is one of the leading causes of preventable deaths and introduced as one of the main risk factors to the development of different types of diseases around the world, especially in relation to non-contagious diseases (like cardiovascular and respiratory diseases, cancer and stroke) [8] [9].

Ketamine is produced as a liquid which can be injected; as a white or off-white powder, which is snorted or dissolved in water and drank; or as a pill. It can be snorted or added to cannabis or cigarettes. It has been used as a date rape drug 
because it is odorless and colorless and is not detected by the victim in a beverage, often rendering its victim completely helpless [10].

The rate of drug abuse and alcohol use among high school students and university students remains unacceptably high. Studies in United States indicate $63.1 \%$ of college students used alcohol in the past 30 days, $16.6 \%$ and $14.5 \%$ of students reported past 30-day use of tobacco and marijuana, respectively [11] [12]. Prevalence of substance abuse among Iranian college students was reported between $4.8 \%$ to $33 \%$ in previous studies [13].

Lifetime prevalence rate of any substance use among college students in western Kenya was $69.8 \%$, and lifetime prevalence rate of alcohol use was 51.9\%, while $97.6 \%$ of alcohol users had consumed alcohol in the week prior to the study [14] [15]. Lifetime prevalence of substance use among medical students in Nigeria was $65 \%$. The most commonly used substances were alcohol $63.4 \%$, mild stimulants $15.6 \%$, tobacco $15 \%$ and sedatives $6.1 \%$ [16].

Globally, cigarette smoking is common among the youths. Student Tobacco use rates are even higher than the corresponding rates in adults in many countries [5]. Most regular smokers initiate smoking before 20 years of age. Youth may have several reasons for starting tobacco use, including looking "cool", "mature", or "sociable", or believing that tobacco use is good for coping with stress and weight control. The factors increasing youth tobacco initiation may vary across countries, but some common factors are: tobacco use by parents or peers; exposure to tobacco advertising; acceptability of tobacco use among peers or in social norms; advertisement in movies or tobacco commercials; having depression, anxiety, or stress; and higher accessibility and lower prices of tobacco products [17].

For some substances, such as tobacco, effects are caused by long-term use. For other drugs, a single use can cause significant disease. In addition to their direct effects on health, drugs produce other indirect effects. Many drugs lessen inhibitions and increase the likelihood that a person will participate in risky behavior. Studies show that the use of alcohol and drugs among teenagers increases chances for teen pregnancy and contracting HIV/AIDS or other sexually transmitted diseases.

Certain risk factors may increase someone's likelihood to abuse substances. Factors within a family that influence a child's early development have been shown to be related to increased risk of drug abuse: Chaotic home environment, Ineffective parenting and lack of nurturing and parental attachment. Factors related to a child's socialization outside the family may also increase risk of drug abuse: Inappropriately aggressive or shy behavior in the classroom, poor social coping skills, poor school performance, association with a deviant peer group, perception of approval of drug use behavior. People take drugs because they want to change something about their lives. Alienation, low motivation, sensation seeking, willingness to take risks and need for stimulation have been associated with drug use [18]. Also associated with substance use are low self-esteem, low self-confidence, need for social approval, high anxiety, low assertiveness, re- 
belliousness, low personal control and low self-efficacy [19]. Such people think that drugs are a solution. Eventually, the drugs become the problem destroying and ruining millions of lives every year [5].

One of the foremost essential steps to combat this challenge is to document the extent, pattern and trends of substance abuse to appreciate the magnitude and severity of the problem [20]. The key risk period for substance use is during major transitions and entering college is one of such. On entering college, adolescents face extra social, emotional and educational challenges. They may also be exposed to greater availability of psychoactive substances and social activities involving substances. As a result, young adult interventions are necessary at this stage of development. By becoming informed about alcohol and other drug prevention efforts on college campuses, prospective college students can increase the likelihood that they will avoid alcohol and other drug-related problems and have their dreams and expectations for college met. Therefore, investigating the situation and knowing present conditions of substance abuse among students is necessary in all steps of planning [3]. People should be asked if they use substances, legal or illegal; they should always be advised to stop; and they should be offered assistance in doing so. Therefore, the information obtained from substance use among nursing students can be used for formulating interventions as well as for educational and prevention programs in high-risk groups

Prevention programs should enhance protective factors and reverse or reduce risk factors and should address the type of drug abuse problem in the local community, target modifiable risk factors, and strengthen identified protective factors [21]. It is essential to reach teenagers and other young smokers with smoking cessation messages and aids. The younger someone is when they stop smoking, the greater the benefit in terms of years of life saved [5].

The objective of this study is to ascertain the prevalence and types of substances used by entering nursing students as a baseline for planning prevention and mental health promotion activities in the college.

\section{Methods}

\subsection{Participants and Procedure}

This cross-sectional study was conducted in 2020 on all first-year nursing students of the College of Nursing and Midwifery Jos, Plateau state, Nigeria. To enroll participants and collect data, they were first educated on the nature and effect of substances of abuse. Their consent for participating in the study was sought and obtained in writing. Also, consent for urine testing was sought and obtained in writing. Confidentiality was explained and ensured on all the personal information given. Sociodemographic data including age, gender, parental history of alcohol use, lifetime history of any substance use was collected using a questionnaire prepared by the researchers.

The study population consisted of 206 freshmen of the College of Nursing and Midwifery Jos, Plateau state, Nigeria. The study protocol was approved by the 
Ethics Committee of Plateau Specialist Hospital. Permission was sought for and obtained from the Principal, School of Nursing, Jos.

Hopkins Symptom Checklist -25 (HSCL-25) was used to elicit symptoms of anxiety and depression among the participants. The anxiety scale has 10 questions (Questions 1 - 10). The depression scale has 15 questions (Questions 11 25). For the responses, each item is assigned the following numbers: $1=$ "Not at all", 2 = "A little", 3 = "Quite a bit"; and 4 = "Extremely". Responses are summed and divided by the number of answered items to generate the anxiety and depression scores respectfully. HSCL-25 is a screening and not a diagnostic instrument for anxiety and depression. Individuals with scores on anxiety (Questions 1 - 10) and/or depression (Question 11 - 25) and/or total (Questions 1 - 25) greater than 1.75 are considered symptomatic. The HSC-25 has been widely used in studies across cultures and languages including Nigeria [22].

Drug of abuse testing was done using a 10-panel test strip of the Drugs of Abuse (DOA) Multi Panel. The Strip utilizes urine samples and tests for the following parameters: THC (Cannabis), COC (Cocaine), COT (Nicotine), MOR (Opioid, Codeine, Pentazocine), BZD (Benzodiazepines), KET (Ketamine), TML (Tramadol), ETG (Alcohol), AMP (Amphetamines), MDMA (Estacy). Results were explained privately to all the participants who authenticated the urine test results. Brief intervention was given to those whose oral history showed significant substance use.

\subsection{Statistical Analysis}

Quantitative variables were expressed as means with SDs, and qualitative/categorical ones as frequencies and percentages. The level of significance was $P<0.05$. Data were analyzed using the SPSS software for Windows, ver. 22.0.

\section{Results}

There were 206 participants comprising 65 males and 141 females. More than half of the participants were between the ages of 20 - 24 years. Their ages ranged from 17 years to 48 years with a mean age of 21.53 years $(S D=3.19)$.

There were 45 students who used substances of various types within the last 30 days of this study, giving a $21.8 \%$ prevalence of any substance use. More females in this study, 33 (23.4\%) were using substances compared to 12 (18.5\%) of males who used substances in the study. Only 9 (4.5\%) of participants admitted to the use of alcohol but denied use of other substances on the self-reported questionnaire. Thirty-two (15.5\%) participants reported that their fathers use alcohol while 4 (4.4\%) reported that their mothers use alcohol also.

Following urine testing, the prevalence of alcohol use was $23(11.2 \%)$, ketamine $13(6.3 \%)$, nicotine $3(1.5 \%)$, benzodiazepines $3(1.5 \%)$, cannabis $2(1.0 \%)$, opioids $1(0.5 \%)$. This pattern was consistent among male and female participants. None of the participants was found with cocaine, heroin or estacy use. Only 4 (17.4\%) of those who use alcohol has a father who uses alcohol also while 
$1(4.3 \%)$ of those who use alcohol has a mother that uses alcohol. None of the sociodemographic variables were significantly associated with having a positive drug screen in the study (Table 1).

Symptoms suggestive of psychological disturbances were high among the participants as $80(38.8 \%)$ had significant total scores on HSCL-25. More than half of the participants 118 (57.3\%) reported significant symptoms of anxiety while $82(39.8 \%)$ of the participants reported significant symptoms of depression. All the participants that use nicotine, opioids and cannabis in the study had no symptomatic anxiety and depression scores. Among those that uses benzodiazepines, $1(2.6 \%)$ and $2(3.8 \%)$ were symptomatic for both anxiety and depression respectfully. Out of those who use alcohol, 19 (26.1\%) and 14 (31.8\%) were symptomatic for anxiety and depression respectfully while 8 (12.7\%) and 7 (18.4\%) of those who uses ketamine had symptomatic anxiety and depression respectfully (Table 2 ).

Alcohol use was significantly related to having symptomatic anxiety $(P=0.02)$ and depression $(P=0.00)$ in the study (Table 3$)$.

Table 1. Demographic and substance use pattern of participants.

\begin{tabular}{|c|c|c|c|c|c|c|c|}
\hline & & BZD & COT & ETG & KET & OPI & THC \\
\hline & Frequency (\%) & YES & YES & YES & YES & YES & YES \\
\hline & & NO & NO & NO & NO & NO & $\mathrm{NO}$ \\
\hline \multicolumn{8}{|l|}{ GENDER } \\
\hline \multirow{2}{*}{ Male } & \multirow{2}{*}{$65(31.6 \%)$} & $1(1.7 \%)$ & $1(2.6 \%)$ & $7(16.3 \%)$ & 3 (7.9\%) & $0(0.0 \%)$ & $0(0.0 \%)$ \\
\hline & & $59(98.3 \%)$ & 37 (97.4\%) & $36(83.7 \%)$ & 35 (92.1\%) & $37(100.0 \%)$ & 37 (100.0\%) \\
\hline \multirow{2}{*}{ Female } & \multirow{2}{*}{$141(68.4)$} & $2(2.6 \%)$ & $2(3.1 \%)$ & $16(21.3 \%)$ & $10(14.5 \%)$ & $1(1.6 \%)$ & $2(3.1 \%)$ \\
\hline & & 75 (97.4\%) & $63(96.9 \%)$ & $59(78.7 \%)$ & $59(85.5 \%)$ & $63(98.4 \%)$ & $63(96.9 \%)$ \\
\hline \multicolumn{8}{|c|}{ AGE GROUP } \\
\hline \multirow{2}{*}{$16-19$} & \multirow{2}{*}{$60(29.1 \%)$} & $1(2.7 \%)$ & $1(3.3 \%)$ & $6(17.6 \%)$ & $4(12.9 \%)$ & $0(0.0 \%)$ & $0(0.0 \%)$ \\
\hline & & $36(97.3 \%)$ & $29(96.7 \%)$ & $28(82.4 \%)$ & $27(87.1 \%)$ & $29(100.0 \%)$ & $29(100.0 \%)$ \\
\hline \multirow{2}{*}{$20-24$} & \multirow{2}{*}{$113(54.0 \%)$} & $2(2.7 \%)$ & $1(3.3 \%)$ & $15(23.8 \%)$ & $8(14.0 \%)$ & $1(1.9 \%)$ & $2(3.7 \%)$ \\
\hline & & $73(97.3 \%)$ & $52(98.1 \%)$ & $48(76.2 \%)$ & $49(86.0 \%)$ & $52(98.1 \%)$ & $52(96.3 \%)$ \\
\hline \multirow{2}{*}{$25-29$} & \multirow{2}{*}{$27(13.1 \%)$} & $0(0.0 \%)$ & $1(6.3 \%)$ & $2(11.8 \%)$ & $1(6.7 \%)$ & $0(0.0 \%)$ & $0(0.0 \%)$ \\
\hline & & $20(100.0 \%)$ & $15(93.8 \%)$ & $15(88.2 \%)$ & $14(93.3 \%)$ & $15(100.0 \%)$ & $15(100.0 \%)$ \\
\hline \multirow{2}{*}{$30-34$} & \multirow{2}{*}{$4(1.9 \%)$} & $0(0.0 \%)$ & $0(0.0 \%)$ & $0(0.0 \%)$ & $0(0.0 \%)$ & $0(0.0 \%)$ & $0(0.0 \%)$ \\
\hline & & $4(100.0 \%)$ & $3(100.0 \%)$ & $3(100.0 \%)$ & $3(100.0 \%)$ & $3(100.0 \%)$ & $15(96.3 \%)$ \\
\hline \multirow{2}{*}{$40-44$} & \multirow{2}{*}{$1(0.5 \%)$} & $0(0.0 \%)$ & $0(0.0 \%)$ & $0(0.0 \%)$ & $0(0.0 \%)$ & $0(0.0 \%)$ & $0(0.0 \%)$ \\
\hline & & $1(100.0 \%)$ & $1(100.0 \%)$ & $1(100.0 \%)$ & $1(100.0 \%)$ & $1(100.0 \%)$ & $3(100.0 \%)$ \\
\hline $45-49$ & $1(0.5 \%)$ & & & & & & \\
\hline \multirow{2}{*}{ TOTAL } & \multirow{2}{*}{$206(100.0 \%)$} & $3(2.2 \%)$ & $3(2.9 \%)$ & $23(19.5 \%)$ & $13(12.1 \%)$ & $1(1.0 \%)$ & $2(2.0 \%)$ \\
\hline & & $134(97.8 \%)$ & $100(97.1 \%)$ & $95(80.5 \%)$ & $94(87.9 \%)$ & $100(99.0 \%)$ & $100(98.0)$ \\
\hline \multicolumn{8}{|c|}{ Father's use of alcohol } \\
\hline YES & 32 & $1(5.6 \%)$ & $1(7.7 \%)$ & $4(26.7 \%)$ & $1(8.3 \%)$ & $0(0.0 \%)$ & $2(2.2 \%)$ \\
\hline NO & $(15.5 \%)$ & $17(94.4 \%)$ & $12(92.3 \%)$ & $11(73.3 \%)$ & $11(100.0 \%)$ & $12(100.0 \%)$ & $97(98.0 \%)$ \\
\hline \multicolumn{8}{|c|}{ Mother's use of alcohol } \\
\hline YES & $4(4.4 \%)$ & $1(14.3 \%)$ & $1(25.0 \%)$ & $1(25.0 \%)$ & $1(33.3 \%)$ & $0(0.0 \%)$ & $0(0.0 \%)$ \\
\hline NO & & $6(85.7 \%)$ & $3(75 \%)$ & $3(75.0 \%)$ & $2(66.7 \%)$ & $3(100.0 \%)$ & $3(100.0 \%)$ \\
\hline
\end{tabular}


Table 2. Prevalence of anxiety and depression symptoms among substance users.

\begin{tabular}{cccc}
\hline SCORE & ANXIETY (HSCL-25) & DEPRESSION (HSCL-25) & TOTAL SCORE (HSCL-25) \\
\hline$<1.75$ & $88(42.7 \%)$ & $124(60.2 \%)$ & $126(61.2 \%)$ \\
$\geq 1.75$ & $118(57.3 \%)$ & $82(39.8 \%)$ & $80(38.8 \%)$ \\
& $206(100.0 \%)$ & $206(100.0 \%)$ & $206(100.0 \%)$ \\
\hline
\end{tabular}

Table 3. Relationship between alcohol use and having symptomatic anxiety or depression.

\begin{tabular}{|c|c|c|c|c|c|c|c|}
\hline & \multicolumn{2}{|c|}{ ALCOHOL } & \multirow{2}{*}{$P$ value } & & \multicolumn{2}{|c|}{ ALCOHOL } & \multirow{2}{*}{$P$ value } \\
\hline & YES & No & & & YES & No & \\
\hline ANXIETY & \multicolumn{7}{|c|}{ DEPRESSION } \\
\hline NO & $4(17.4 \%)$ & $41(43.2 \%)$ & 0.02 & NO & $9(39.1 \%)$ & $65(68.4 \%)$ & 0.00 \\
\hline YES & $19(82.6 \%)$ & $54(56.8 \%)$ & & YES & $14(60.9 \%)$ & $44(37.3 \%)$ & \\
\hline TOTAL & $23(100.0 \%)$ & $95(100.0 \%)$ & & TOTAL & $23(100.0 \%)$ & $95(100.0 \%)$ & \\
\hline
\end{tabular}

\section{Discussion}

The finding of high lifetime prevalence of any substance use among the new college students is an indication that substance use is common among the nursing students. This is an agreement with other studies carried out among college students in and around the world and emphasizes the need to have a deliberate action against substance abuse among students [1] [2] [3] [4] [16]. Substance use in the study was more prevalent among those aged $20-29$ years. This is in keeping with several other studies [16] [23]. The age range corresponds to age of freedom from parental monitoring and autonomy towards decision concerning an individual mostly in Nigeria. Only few participants willingly admitted that they use alcohol. This could be due to the reason that they were not comfortable with their habit despite the fact that alcohol use in Nigeria is legal. The fact that all the first-year students denied other substance use indicates the knowledge that use of illegal substances is wrong and is an abuse. The discovery of higher rate of substance use from urine testing compared to volunteered information underscores a need to use urine test in confirming substance abuse whenever suspicion arises. Compulsory urine testing at college entry is practicable. It will serve as a baseline for comparison of future findings in an individual while in school. It could serve as a pointer to substance use disorder and inform need for intervention. It gives a window into the trend of substance use in the larger community and influence opinion and strategies towards combating the menace. However, it stands the risk of giving a false positive result, exploitation of parents and students, and could even stimulate desire for substance use behavior in naïve individuals.

Alcohol was the most used substance in the study. This is consistent with findings across literature [11] [12] [16] [23] [24] [25] [26]. The discovery that more females than males were using substance is alarming. This finding was 
consistent among all the substances reported but differed with other findings in and around the country [16] [23] [24] [25] [26]. However, this could be due to the fact that in the population studied, the number of females was more than twice that of males. Ketamine use was equally high suggesting that pattern of substance use in and around Jos could be changing. This has been observed in other studies [23]. Ketamine abuse in the study is more worrisome considering the fact that it was higher among females. This is in view of the fact that ketamine could be used as a date rape drug. Also worrisome was that during de-briefing of the test results, all the participants denied knowledge of having used ketamine in the 30 days preceding the study suggesting the possibility of consuming the ketamine laced inside other substances that were used. There is also a probability of ketamine finding in urine testing being a false positive, hence the need for further research into the finding.

Few participants reported alcohol use among their parents however, there was no statistical correlation between parental use of alcohol and respondents use of all the substances. This agrees with previous findings on influence of parental substance use [25]. A high rate of anxiety and depressive symptoms among the participants were an indication of the level of psychological disturbance. The finding that a large number of those with psychological disturbance use drugs could indicate self-medication. Self-medication with substances is well documented in literatures. Alcohol was the only substance that statistically correlated with having symptomatic anxiety and depression in the study. This could suggest the probability of anxiety and depression being causes of alcohol use in the study. One danger of higher number of females using alcohol is having unprotected sex while under the influence of alcohol or being too drunk and not knowing whether they consented to sexual intercourse. According to National Institute on Alcohol Abuse and Alcoholism (NIAA), 400,000 students in America had unprotected sex while under the influence of alcohol [27].

The absence of cocaine, heroin and estacy use among the students could either suggest unavailability of these substances around the college or lack of indulgence due to high cost of the mentioned substances.

\section{Recommendations}

Considering the high prevalence of substance use especially alcohol and ketamine, it essential to design educational interventions to prevent substance abuse and dependence in the college, paying attention to predictive factors among college students. Mandatory drug testing at college entry should be considered as a baseline. Intermittent testing of suspected cases of disorder should be provided for early detection of substance use disorder and intervention. This is practicable as it does not require much expertise, gives result fast and is relatively cost effective. School based prevention curriculum and activities based on generic self-management and social skills should be designed to prevent the initiation of drug use and also prevent problems that may arise from drug use. Preventive strategies are usually more effective if implemented at specific periods before the 
onset of mental disorder, in order to be maximally effective.

Drug free clubs owned and manned by the students should be encouraged in the campus. This should serve as a good source of peer education and training. It will also provide information and advice on promotion of good mental health to current users to reduce drug related problems to themselves and others. Mental health promotion involves promoting the value for mental health and improving the coping capacities of individuals rather than amelioration of symptoms and deficits. Peer initiatives are more interactive and efficacious especially if carried out in non-formal settings. The drug free clubs should also provide a wide range of drugs and alcohol-free social and recreational activities in the campus.

Forming partnership with the local communities to ensure that alcohol is not served to intoxicated students would also be vital. Strengthening their academic activities, keeping the library and recreational facilities well equipped with proper mental health promotion materials would be of great importance.

\section{Acknowledgements}

We acknowledge the permission and support of Plateau state Health Management Board and the Provost of the Plateau State College of Nursing and Midwifery towards the actualization of this research work.

\section{Conflicts of Interest}

We declare that there was no conflict of interest with respect to this research work.

\section{Authors' Contribution}

Author A and B designed the study, wrote the protocol and the first draft of the manuscript. Author C and D managed the analysis of the study. Author A, E and $\mathrm{F}$ managed the literature searches. All authors read and approved the final manuscript.

\section{References}

[1] U.S. Department of Education, Office of Safe and Drug-Free Schools (2008) Alcohol and other Drug Prevention on College Campuses: Model Programs. Washington, DC. http://www.ed.gov

[2] Jalilian, F., Karami Matin, B., Ahmadpanah, M., Ataee, M., Ahmadi Jouybari, T., Eslami, A.A. and Mirzaei Alavijeh, M. (2015) Socio-Demographic Characteristics Associated with Cigarettes Smoking, Drug Abuse and Alcohol Drinking among Male Medical University Students in Iran. Journal of Research in Health Sciences, $15,42-46$.

[3] Wechsler, H., Lee, J.E., Kuo, M. and Lee, H. (2000) College Binge Drinking in the 1990s: A Continuing Problem: Results of the Harvard School of Public Health 1999 College Alcohol Study. Journal of American College Health, 48, 199-210. https://doi.org/10.1080/07448480009599305

[4] Paschall, M.J., Ringwalt, C., Wyatt, T. and DeJong, W. (2014) Effects of an Online Alcohol Education Course among College Freshmen: An Investigation of Potential 
Mediators. Journal of Health Communication, 19, 392-412. https://doi.org/10.1080/10810730.2013.811328

[5] Tarbish, S.A., Nabil, S., Nazira, S. and Pervaiz, S.S. (2015) Substance Use Disorder: A Cultural Catastrophe. International Journal of Science and Research, 4, 1539-1563.

[6] Rehm, J., Gmel Sr, G.E., Gmel, G., Hasan, O.S., Imtiaz, S., Popova, S., Probst, C., Roerecke, M., Room, R. and Samokhvalov, A.V. (2017) The Relationship between Different Dimensions of Alcohol Use and the Burden of Disease-An Update. Addiction, 112, 968-1001. https://doi.org/10.1111/add.13757

[7] Hingson, R., Heeren, T., Winter, M. and Wechsler, H. (2005) Magnitude of Alcohol-Related Mortality and Morbidity Among U.S. College Students Ages 18-24: Changes from 1998 to 2001. Annual Review of Public Health, 26, 259-279. https://doi.org/10.1146/annurev.publhealth.26.021304.144652

[8] Haenle, M.M., Brockmann, S.O., Kron, M., Bertling, U., Mason, R.A., Steinbach, G. and Kratzer, W. (2006) Overweight, Physical Activity, Tobacco and Alcohol Consumption in a Cross-Sectional Random Sample of German Adults. BMC Public Health, 6, Article No. 233. https://doi.org/10.1186/1471-2458-6-233

[9] Moeini, B., Poorolajal, J. and Gharlipour, Z. (2012) Prevalence of Cigarette Smoking and Associated Risk Factors among Adolescent in Hamadan City in 2010. Journal of Research in Health Sciences, 12, 31-37.

[10] Ketamine Addiction and Abuse-Hallucinogenic Addiction (2020) Addiction Centre. https://www.addictioncenter.com/drugs/hallucinogens/ketamine

[11] American College Health Association (2009) National College Health Assessment Spring 2008 Reference Group Data Report (Abridged): The American College Health Association. Journal of American College Health, 57, 477-488. https://doi.org/10.3200/JACH.57.5.477-488

[12] Hingson, R.W., Zha, W. and Weitzman, E.R. (2009) Magnitude of and Trends in Alcohol-Related Mortality and Morbidity among US College Students Ages 18-24, 1998-2005. Journal of Studies on Alcohol and Drugs, Supplement, 16, 12-20. https://doi.org/10.15288/jsads.2009.s16.12

[13] Ahmadi, J. and Hasani, M. (2003) Prevalence of Substance Use among Iranian high School Students. Addictive Behaviors, 28, 375-379. https://doi.org/10.1016/S0306-4603(01)00246-5

[14] Atwoli, L and Mungla, P.A., Ndung'u, M.N., Kinoti, K.C. and Ogot, E.M. (2011) Prevalence of Substance Use among College Students in Eldoret, Western Kenya. BMC Psychiatry, 11, Article No. 34. https://doi.org/10.1186/1471-244X-11-34

[15] Partanen, J. (1993) Failures in Alcohol Policy: Lessons from Russia, Kenya, Truk and history. Addiction, 88, 129S-134S.

[16] Babalola, E.O., Akinhami, A. and Ogunwale, A. (2014) Who Guards the Guards: Drug Use Pattern among Medical Students in a Nigerian University. Annals of Medical and Health Science Research, 4, 397-403. https://doi.org/10.4103/2141-9248.133467

[17] Eriksen, M.P., Judith, M., Neil, S., Farhad, S. and Jeffrey, D. (2015) The Tobacco Atlas. 5th Edition, American Cancer Society, New York.

[18] Hawkins, J.D., Lishner, D.M., Jenson, J.M. and Catalano, R.F. (1987) Delinquents and Drugs: What the Evidence Suggests about Prevention and Treatment Programming. In: Brown, B.S. and Mills, A.R., Eds., Youth at High Risk for Substance Abuse, National Institute on Drug Abuse, Rockville, 81-131.

[19] Schinke, S.P., Botvin, G.J. and Orlandi, M.A. (1991) Substance Abuse in Children 
and Adolescents: Evaluation and Intervention. Sage Publications, Newbury Park.

[20] Basu, D., Aggarwal, M., Partha, P.D., Mattoo, S.K., Parmanand, K. and Vijoy, K.V. (2012) Changing Pattern of Substance Abuse in Patients Attending a De-Addiction Center in North India (1978-2008). Indian Journal of Medical Research, 135, 830-836.

[21] Hawkins, J.D., Catalano, R.F. and Arthur, M. (2002) Promoting Science-Based Prevention in Communities. Addictive Behaviors, 90, 1-26.

[22] Nwoga, C.N., Dakwak, S.J., Agbir, T.M., Audu, M.D., Goar, S.G., Tungchama, F.P., et al. (2018) Functional Disability and Depression among Internally Displaced persons in North-East, Nigeria. Journal of Biomedical Research \& Clinical Practice, 1 , 47-56. https://doi.org/10.46912/jbrcp.34

[23] Nwoga, C.N., Ndak, A.Z., Audu, M.D., Goar, S.G., Armiya'u, A.Y., Agbir, T.M and Dakwak, S.J. (2019) Pattern and Effect of Substance Use in Jos South, North Central Nigeria. Journal of Research in Basic \& Clinical Sciences, 1, No. 2.

[24] Ohaeri, J.U. and Odejide, O.A. (1993) Admissions for Drug and Alcohol Related Problems in Nigeria Psychiatric Care Facilities in One Year. Drug and Alcohol Dependence, 3, 101-109. https://doi.org/10.1016/0376-8716(93)90062-U

[25] Onoja, M.O. (2020) Prevalence of Substance Abuse among Secondary School Students-A Comparative Study of Government and Private Secondary Schools in Jos, Nigeria. National Institute on Drug Abuse. Available online on NIDA International Drug Abuse Research Abstract Database.

[26] Isah, H.O., Basi, A.P. and Chima, G. (2017) Substances in Use and Abused by Adolescent Population of Senior Secondary Schools in Jos South LGA, Plateau State, Nigeria. Journal of Pharmaceutical and Allied Sciences, 14, No. 3.

[27] National Institutes of Health (2002) Task Force of the National Advisory Council on Alcohol Abuse and Alcoholism, National Institute on Alcohol Abuse and Alcoholism. A Call to Action: Changing the Culture of Drinking at U.S. Colleges. National Institutes of Health, Washington DC.

http://www.collegedrinkingprevention.gov/StatsSummaries/snapshot.aspx 